\title{
Recurso terapêutico ocupacional para tratamento de delirium em pacientes com COVID-19
}

\author{
Occupational therapeutic resource for treating delirium in \\ patients with COVID-19
}
Recurso terapéutico ocupacional para el tratamiento del delirio en pacientes con COVID-19

\author{
Renata Gomes Barreto ${ }^{1}$, Wendy Chrystyan Medeiros de Sousa ${ }^{2}$, \\ Silmara Maria Alves Fernandes da Silva ${ }^{3}$, Thais Alves de Souza ${ }^{4}$, \\ Erika Carneiro da Silva ${ }^{5}$, Bruno da Silva Brito ${ }^{6}$, Gilberto Costa \\ Teodozio7, Maria Cecília de Araújo Silvestre ${ }^{8}$
}

\begin{abstract}
1.Terapeuta Ocupacional, Pós Graduada em Gerontologia (CINTEP), Especialista em Qualidade em Saúde e Segurança do Paciente (FIOCRUZ), Coordenadora da Terapia Ocupacional do Hospital Metropolitano Dom José Maria Pires, Santa Rita-PB, Brasil. ORCID: https://orcid.org/0000-0001-7166-9674

2.Terapeuta Ocupacional, Pós Graduada em Gerontologia (CINTEP), Mestranda em Gerontologia (UFPB), Terapeuta Ocupacional do Hospital Metropolitano Dom José Maria Pires, Santa Rita-PB, Brasil. ORCID: https://orcid.org/0000-0002-9761-9488

3.Terapeuta Ocupacional, Residência Multiprofissional em Saúde Mental (UFPB), Terapeuta Ocupacional do Hospital Metropolitano Dom José Maria Pires, Santa Rita-PB, Brasil. ORCID: https://orcid.org/00000003-3280-6482

4.Terapeuta Ocupacional, Residência Multiprofissional em Saúde Hospitalar Criança e Adolescente (UFPB), Terapeuta Ocupacional do Hospital Metropolitano Dom José Maria Pires, Santa Rita-PB, Brasil. ORCID: https://orcid.org/0000-0002-6688-9266

5.Terapeuta Ocupacional, Pós Graduanda em Gestão em Saúde Pública (Universidade Estácio de Sá), Terapeuta Ocupacional do Hospital Metropolitano Dom José Maria Pires, Santa Rita-PB, Brasil. ORCID: https://orcid.org/0000-0003-3818-5410

6. Fisioterapeuta, Mestre em Neurociência Cognitiva e Comportamento (UFPB), Especialista em Qualidade em Saúde e Segurança do Paciente (FIOCRUZ), Gerente Multidisciplinar e de Qualidade do Hospital Metropolitano Dom José Maria Pires, Santa Rita-PB, Brasil. ORCID:. https://orcid.org/0000-0001-5040997X

7.Enfermeiro, Especialista em Enfermagem Dermatológica (Faculdade Estácio de Sá), Especialista Gestão Hospitalar (Faculdade Uniasselvi), Diretor Assistencial do Hospital Metropolitano Dom José Maria Pires, Santa Rita-PB, Brasil. ORCID: https://orcid.org/0000-0002-5200-4658

8.Terapeuta Ocupacional, Mestre em Ciências da Reabilitação (UFRN), Doutoranda em Neurociência Cognitiva e Comportamento (UFPB), Terapeuta Ocupacional do Hospital Metropolitano Dom José Maria Pires, Santa Rita-PB, Brasil. ORCID: https://orcid.org/0000-0002-0687-8418
\end{abstract}

\section{Resumo}

Objetivo. A reabilitação cognitiva precoce e suas formas de intervenção para prevenção e tratamento do delirium é um foco relativamente novo de pesquisa. O objetivo deste artigo é apresentar uma alternativa de intervenção cognitiva para prevenção e manejo de delirium em pacientes com COVID-19 no contexto hospitalar. Método. Trata-se de um estudo descritivo definido pela elaboração de um livro como recurso terapêutico ocupacional baseado na intervenção cognitiva para prevenção e manejo de delirium dos pacientes internados em um hospital estadual de referência no tratamento do COVID-19. Resultados. O livro interativo "Você pode tudo menos desistir" utilizou estratégias de compensação que facilitam e fortalecem o processamento cognitivo, além de treinamento cognitivo de funções específicas, tais como a memória, pensamento, aspectos emocionais e consciência da própria identidade. Conclusão. Sugere-se que o livro interativo pode ser utilizado como uma estratégia não farmacológica de intervenção, proporcionando o engajamento terapêutico durante as atividades de estimulação cognitiva para prevenção e manejo de delirium em pacientes hospitalizados com COVID-19.

Unitermos. Infecções por Coronavirus; Cognição; Delirium; Terapia Ocupacional 


\begin{abstract}
Objective. Early cognitive rehabilitation and its forms of intervention for the prevention and treatment of delirium is a relatively new focus of research. The aim of this article is to present an alternative cognitive intervention for the prevention and management of delirium in patients with COVID-19 in the hospital context. Method. This is a descriptive study defined by the elaboration of a book as an occupational therapeutic resource based on the cognitive intervention for the prevention and management of delirium of patients hospitalized in a public hospital of reference in the treatment of COVID-19. Results. The interactive book "You can do anything but give up" used compensation strategies that facilitate and strengthen cognitive processing, in addition to cognitive training of specific functions, such as memory, thinking, emotional aspects and awareness of self-identity. Conclusion. It is suggested that the interactive book can be used as a non-pharmacological intervention strategy, providing therapeutic engagement during cognitive stimulation activities for the prevention and management of delirium in patients hospitalized with COVID-19.
\end{abstract}

Keywords. Coronavirus Infections; Cognition; Delirium; Occupational Therapy

\title{
Resumen
}

Objetivo. La rehabilitación cognitiva temprana y sus formas de intervención para laprevención y eltratamientodeldelirio es un enfoque relativamente nuevo de investigación.Presentar una alternativa de intervención cognitiva para laprevención y eltratamientodeldelirioen pacientes con COVID-19 enel contexto hospitalario. Método. Este esunestudiodescriptivo definido por eldesarrollo de un libro como recurso terapéutico ocupacional basadoenlaintervención cognitiva para laprevención y tratamientodeldelirioen pacientes admitidos enun hospital estatal de referencia eneltratamiento de COVID-19. Resultados. El libro interactivo "Lopuedes todo menos rendirte" utilizóestrategias de compensación que facilitan y fortalecenelprocesamiento cognitivo, ademásdelentrenamiento cognitivo de funciones específicas, tales como memoria, pensamiento, aspectos emocionales y conciencia de lapropiaidentidad. Conclusión. Se sugiere que se pueda utilizar el libro interactivo como una estrategia no farmacológica de intervención, ofreciéndolescompromisoterapéutico durante lasactividades de estimulación cognitiva para laprevención y eltratamientodeldelirio a pacientes hospitalizados con COVID-19.

Palabras clave. Infecciones por Coronavirus; Cognición; Delirio; Terapia Ocupacional

Trabalho realizado no Hospital Metropolitano Dom José Maria Pires, Santa Rita-PB, Brasil.

\section{INTRODUÇÃO}

Desde dezembro de 2019, múltiplos casos de pneumonia inexplicável foram sucessivamente relatados em alguns hospitais na cidade de Wuhan na China. O patógeno, um novo coronavírus (SARS-CoV-2), foi identificado por 
hospitais locais utilizando o mecanismo de vigilância para "pneumonia de etiologia desconhecida"1.

Os estudos sobre o COVID-19 têm descrito manifestações clínicas típicas incluindo febre, tosse, diarreia e fadiga ${ }^{2}$, porém muitos pacientes hospitalizados podem apresentar episódios de delirium ${ }^{3}$.

Em pacientes com COVID-19, o delirium pode ser uma manifestação de invasão direta do sistema nervoso central (SNC), efeito secundário de falha de outros órgãos, efeito das estratégias sedativas, tempo prolongado de ventilação mecânica ou fatores ambientais, incluindo isolamento social ${ }^{3}$.

A Classificação Internacional de Doenças (CID-10) descreve o delirium como uma síndrome cerebral orgânica com etiologia inespecífica, caracterizada por distúrbios da consciência, atenção, percepção, pensamento, memória, comportamento psicomotor, emoções e ciclo sono-vigília4.

O comprometimento da função cognitiva comumente é associado ao delirium e está relacionado a piores resultados funcionais e maior índice de mortalidade. $O$ tratamento do delirium é desafiador e amplamente preventivo ${ }^{5}$, as evidências apontam para os benefícios de intervenções não farmacológicas para a prevenção e o manejo do delirium.

As intervenções cognitivas são estratégias baseadas em evidências que visam domínios cognitivos afetados pelo delirium, como orientação, memória, pensamento abstrato e função executiva, e podem estimular a plasticidade 
neuronal, melhorando a função cognitiva em pacientes críticos com ou em risco de desenvolver delirium ${ }^{6}$.

A reabilitação cognitiva precoce e suas formas de intervenção para prevenção e tratamento do delirium é um foco relativamente novo de pesquisa. $O$ objetivo deste artigo é apresentar uma alternativa de intervenção cognitiva para prevenção e manejo de delirium em pacientes com COVID-19 no contexto hospitalar.

\section{MÉTODO}

Trata-se de um estudo descritivo com abordagem qualitativa, definido pela elaboração de um recurso terapêutico ocupacional baseado na intervenção cognitiva para prevenção e manejo de delirium dos pacientes internados em um hospital referência estadual no tratamento do COVID-19.

O Livro Interativo "Você pode tudo menos desistir", pertencente a Coletânea "Estimula Mente", foi desenvolvido pela equipe de Terapia Ocupacional da instituição, considerando os aspectos e componentes comuns ao cotidiano dos indivíduos.

O recurso terapêutico, na forma de livro interativo, foi desenvolvido para que suas atividades auxiliem na prevenção e no manejo de delirium em pacientes com COVID-19 levando em consideração os impactos causados pelo processo de hospitalização que acaba acarretando no isolamento e distanciamento dos familiares e na ruptura dos seus papéis ocupacionais. 
Tem como objetivos auxiliar no controle do pensamento com experiência de si e do tempo, possibilitar a evocação de memórias significativas ao indivíduo e as suas atividades diárias, proporcionar novas abordagens sobre temas cotidianos como os sentimentos, medos, sonhos, emoções, auto expressão, opiniões próprias, consciência da realidade e perspectivas futuras, estimular a criatividade, orientação temporal e espacial. Além disso, as atividades auto expressivas do livro visam minimizar o impacto do processo de hospitalização e amenizar a ansiedade que o processo de internação por COVID-19 gera nos pacientes.

O design utilizado para a confecção do livro (Figura 1) seguiu os critérios do Design Universalp e foi baseado em elementos e fatores que favorecem possibilidades de acessibilidade para que o produto seja utilizado pela maioria dos pacientes internos. Para a concepção de um produto funcional, fora determinado as etapas de desenvolvimento até o processo de aplicabilidade durante as intervenções terapêuticas ocupacionais ${ }^{7}$.

O projeto de desenvolvimento do produto incluiu a análise de diversos fatores, como definição de cores e gráficos, materiais de baixo custo, nível de escolaridade e aspectos cognitivos e sensoriais preservados do paciente, entre outros. No entanto, optou-se por utilizar abordagem de conteúdo baseado em elementos gráficos interativos e personalizados de acordo com o contexto atual, com o objetivo de proporcionar uma maior interação e 
engajamento do leitor/paciente na atividade proposta e ganhos funcionais referentes ao desempenho ocupacional.

Figura 1. Capa do livro interativo "Você pode tudo menos desistir".

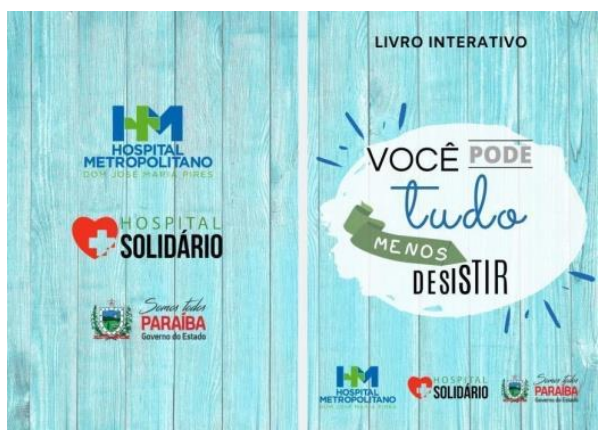

O conteúdo e gráfico interativo foram construídos em uma dimensão para tornar-se atrativo e interessante, com design reflexivo e expressivo, utilizando aspectos relacionados a experiência de si e atividades cotidianas. Além de proporcionar orientações quanto ao uso do recurso durante o período de internação (Figura 2) e estímulos educativos de higienização (Figura 3) que remetem ao contexto atual da pandemia de COVID-19.

As atividades e seus respectivos objetivos foram baseadas no documento da Associação Americana de Terapia Ocupacional "Estrutura da prática da Terapia Ocupacional: domínio e processo"8.

\section{RESULTADOS}

A construção de todas as etapas Livro Interativo "Você pode tudo menos desistir" foi realizada por uma equipe de terapeutas ocupacionais que atuam junto a pacientes com 
COVID-19 em um hospital de referência no estado da Paraíba - Brasil.

Figura 2. Orientações quanto ao uso do recurso.

\section{ORIENTAÇÕES}

Este livro contém 29 páginas, sendo cada

1 uma delas destinada a uma atividade diferente. Durante seu periodo conosco, faremos UMA atividade por dia.

Registre seus dados na página seguinte e reserve um tempo diário para a construção desse livro.

Não esqueça que esse é um momento para 3 extravasar suas idéias e pensamentos. Sabemos que não é fácil, portanto aproveite.

No decorrer dos dias, uma das nossas terapeutas ocupacionais irá acompanhar você nessa jornada. Qualquer dúvida ou 4 esclarecimento, solicite para que um dos responsáveis do setor entre em contato com nossa equipe.

Figura 3. Estímulos educativos de higienização

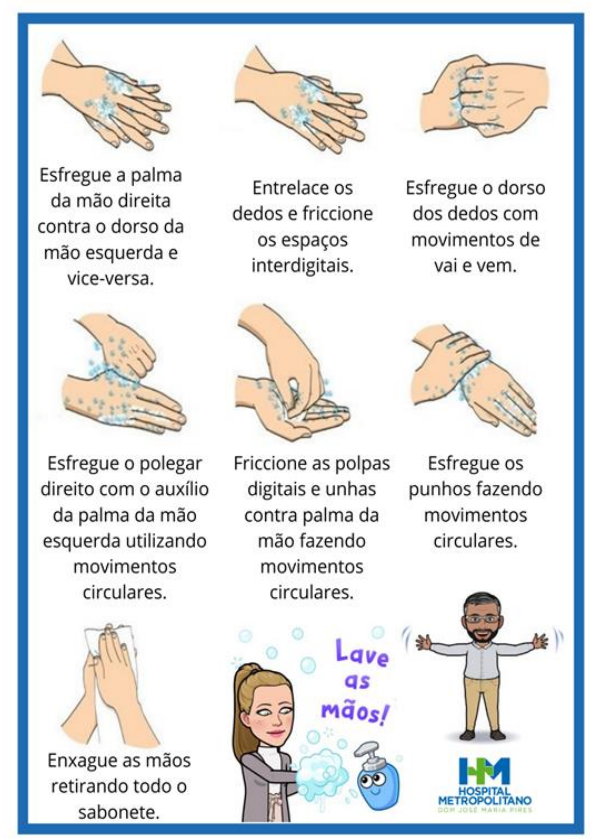


O livro contém 21 atividades (Figura 4) com estímulos e objetivos variados (Tabela 1 ) previamente estabelecidos com foco na prevenção e manejo de delirium em paciente hospitalizados com COVID-19.

Figura 4. Algumas das atividades do Livro Interativo "Você pode tudo menos desistir".
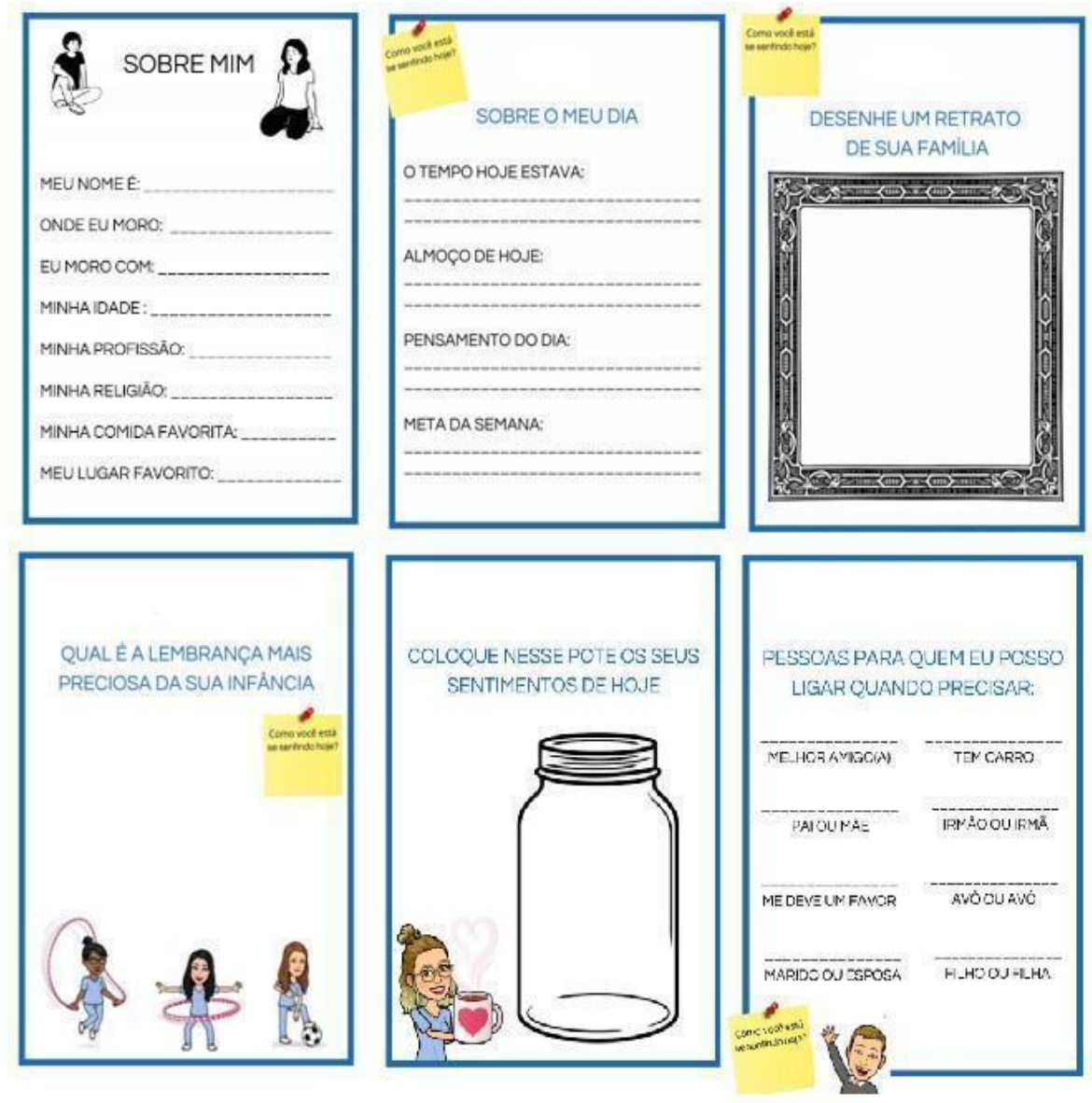
Tabela 1. Objetivos das atividades do livro interativo.

ATIVIDADE

Sobre mim

Pote dos sentimentos

Liste 5 lugares que você queria estar agora

Desenhe um retrato de sua família

Qual é a lembrança mais preciosa da sua infância

Escreva uma carta para alguém que você ama

Escreva uma música que...

Complete a música

Checklist para o futuro

Sobre o meu dia

Imagine que de onde você está tem uma janela. Descreva ou desenho o que você está vendo agora

Agora descreva ou desenhe o que você gostaria de ver na janela

Em uma escala de 1 a 10 o quão feliz você está hoje? Cite 10 coisas em que você é muito
bom

Pessoas para quem eu posso ligar quando precisar
OBJETIVO

Traçar um perfil ocupacional resumido do paciente, através de coleta de informações sobre a pessoa, ocupações, crenças e ambiente do cotidiano do paciente.

Estimular as funções mentais específicas relacionadas às emoções e funções executivas de regulação emocional necessárias para favorecer participação social e uma boa qualidade de vida.

Identificar locais de interesse e possíveis ocupações significativas através dos ambientes de preferência do paciente, estimular funções mentais específicas relacionadas à memória de longo prazo.

Estimular funções mentais específicas relacionadas à memória de longo prazo e funções mentais globais relacionadas a orientação e auto expressão.

Estimular funções mentais específicas relacionadas à memória de longo prazo, lógica, pensamento coerente e consciência da própria identidade.

Estimular funções mentais específicas relacionadas ao alto nível cognitivo, memória, controle do pensamento, consciência da realidade, pensamento coerente e labilidade emocional.

Estimular funções mentais específicas relacionadas ao alto nível cognitivo, memória de curto e longo prazo e funções mentais globais relacionadas ao temperamento e personalidade (auto expressão).

Estimular funções mentais específicas relacionadas ao alto nível cognitivo, atenção, memória, percepção e pensamento coerente.

Estimular funções mentais globais relacionadas a consciência, abertura a experiências, auto expressão e motivação.

Estimular funções mentais específicas relacionadas a memória de curto prazo, controle do pensamento, consciência da realidade versus delírios, lógica e pensamento coerente.

Estimular funções mentais específicas relacionadas a consciência da realidade versus delírios, alto nível cognitivo, lógica e pensamento coerente e funções mentais globais relacionadas a auto expressão

Estimular funções mentais específicas relacionadas a memória de curto prazo, consciência da realidade versus delírios, lógica e pensamento coerente e funções mentais globais relacionadas a auto expressão

Estimular funções mentais específicas relacionadas a regulação da variação das emoções e funções mentais globais relacionadas a estabilidade emocional e a motivação.

Estimular funções mentais específicas relacionadas a consciência da realidade versus delírios, lógica e pensamento coerente e funções mentais globais relacionadas a auto expressão e consciência da própria identidade.

Estimular funções mentais específicas relacionadas a memória e funções mentais globais relacionadas a orientação. 
Tabela 1 (cont.). Objetivos das atividades do livro interativo.

\section{ATIVIDADE}

Quais são os seus desejos para o ano que vem?

Favoritos, circule o seu

Quais os ingredientes são necessários para preparar um bolo

Escreva três coisas que mudaria em você

Libere sua criatividade e utilize os lápis de cor

Descreva o que sentiu durante esse período aqui conosco

Qual a primeira coisa que irá fazer quando sair daqui?

\section{OBJETIVO}

Estimular funções mentais globais relacionadas a auto expressão, desejo e motivação.

Estimular funções mentais globais relacionadas a temperamento e personalidade e desejo.

Estimular funções mentais específicas relacionadas a memória.

Estimular funções mentais específicas relacionadas a consciência da realidade versus delírios, lógica e pensamento coerente, aspectos emocionais e funções mentais globais relacionadas a auto expressão e consciência da própria identidade.

Estimular funções mentais específicas relacionadas a atenção e concentração, funções mentais globais relacionadas a auto expressão e funções neuromusculoesqueléticas relacionadas ao controle motor fino

Estimular funções mentais específicas relacionadas a memória, lógica e pensamento coerente e aspectos emocionais, e funções mentais globais relacionadas a auto expressão, motivação e orientação.

Estimular funções mentais globais relacionadas a consciência, abertura a experiências, auto expressão, desejo e motivação.

As atividades devem ser desenvolvidas de acordo com a avaliação terapêutica ocupacional realizada previamente à aplicação do livro aos pacientes com COVID-19 e podem ser graduadas ou adaptadas conforme a necessidade e condição clínica do paciente.

O livro interativo oferece estratégias de compensação que facilitam e fortalecem o processamento cognitivo, além de treinamento cognitivo de funções específicas, tais como a memória, pensamento, aspectos emocionais e consciência da própria identidade, que podem ser comprometidas em 
pacientes com COVID-19 que apresentem episódios de delirium.

\section{DISCUSSÃO}

O livro interativo "Você Pode Tudo Menos Desistir" pode ser utilizado como uma estratégia centrada no paciente, proporcionando 0 engajamento terapêutico durante as atividades de estimulação cognitiva para prevenção e manejo de delirium em pacientes hospitalizados com COVID-19.

Estudos afirmam que atividades de orientação temporal e espacial, o engajamento terapêutico através de atividades significativas para o paciente e a estimulação de memórias de curto e longo prazo, auxiliam na prevenção e manejo da incidência de episódios de delirium9 .

Muitos pacientes em processo de hospitalização podem desenvolver comprometimentos cognitivos devido aos episódios de delirium e declínio funcional na realização das suas atividades cotidianas, incluindo pacientes com COVID19 como já foi referenciado anteriormente, portanto, o terapeuta ocupacional pode tornar-se essencial na composição da equipe multidisciplinar para contribuir com as suas intervenções.

A intervenção tardia ou ausência de gerenciamento sistematizado para prevenir ou diminuir o delirium durante a hospitalização terá grande impacto na saúde e qualidade de vida do paciente, tendo como resultados o aumento da comorbidade e mortalidade, além do prolongamento da 
internação com comprometimento cognitivo e funcional. Na equipe multidisciplinar no combate ao delirium, a intervenção precoce e intensiva da Terapia Ocupacional em pacientes hospitalizados reduziram a incidência de delirium e dias de internação ${ }^{10}$, além de favorecer a independência funcional nas Atividades de Vida Diária (AVD), prevenção de deterioração motora, cognitiva e social do paciente e auxiliando na diminuição do tempo e efeitos negativos da internação ${ }^{11}$.

Os terapeutas ocupacionais utilizam de atividades significativas que busquem a restauração da rotina diária do sujeito, oferecendo estímulo cognitivo, ajudando assim os clientes a manterem a função e um bom desempenho ocupacional.

As atividades utilizadas devem ser significativas para 0 paciente, coerentes com seus interesses e com seus papéis ocupacionais. O terapeuta ocupacional deve realizar a análise das atividades nas quais o sujeito apresenta demandas para intervenção e considerar a influência de fatores pessoais e ambientais no desempenho ocupacional12. De acordo com a Associação Americana de Terapia Ocupacional, ao utilizar abordagens cognitivas, 0 terapeuta ocupacional promove a estimulação das funções mentais específicas e globais que favorecem as habilidades de desempenho, especificamente as habilidades processuais que são mais afetadas em pacientes com 0 delirium e que, consequentemente acarretam em prejuízo nas demais áreas de desempenho ocupacional ${ }^{8}$. 
O livro interativo pode fornecer através das atividades o desempenho das habilidades processuais que incluem atenção, memória, concentração, sequenciamento, iniciativa, organização, tomada de decisões, estímulos necessários que tem potencial de auxiliar na prevenção e diminuição da incidência de delirium em pacientes hospitalizados com COVID-19.

Há evidências sobre uma incidência reduzida de delirium após a implementação de um protocolo de intervenção cognitiva liderada por terapeutas ocupacionais $^{13}$. As intervenções de treinamento e estimulação cognitiva podem se sobrepor e incluem treinamento de memória usando imagens visuais e treinamento metacognitivo, usando abordagens de autoconsciência e auto regulação para recuperar o funcionamento executivo e diminuir a incidência de delirium ${ }^{14}$.

As atividades do livro foram planejadas e selecionadas considerando a fase do desenvolvimento da adultez, as alterações cognitivas manifestadas durante episódios de delirium junto a pacientes com COVID-19, e utilizando linguagem de fácil compreensão. Ao realizar a intervenção cognitiva, aplicando o livro interativo como recurso, o terapeuta pode graduar a atividade conforme a demanda, pois sua expertise permite adaptar a intervenção centrada no perfil ocupacional e habilidades do indivíduo.

O terapeuta ocupacional é competente para identificar as limitações relacionadas à participação ativa na atividade 
e direcionar estratégias para solucioná-las a partir de sua habilidade em realizar a análise da atividade. Em seu estudo o autor evidencia que o terapeuta ocupacional pode auxiliar para minimizar os impactos negativos oriundos da internação nas demandas motoras, desorientação, confusão mental temporária e estimular para independência e autonomia nas atividades de vida diária ${ }^{15}$.

O livro contém atividades que devem promover orientação espacial para auxiliar o paciente a situar-se e orientar-se, em relação aos objetos, às pessoas e o seu próprio corpo em um determinado espaço, bem como promoção de orientação temporal para auxiliar na noção de tempo durante o afastamento da rotina cotidiana e dos papéis ocupacionais que processo de hospitalização por COVID-19 gera.

Há evidências de uma redução significativa na ocorrência de delirium após a introdução de um protocolo de estimulação cognitiva que incluiu intervenções de orientação ambientais, acústicas e visuais ${ }^{16}$. A falta de orientação espacial e temporal do paciente, representa um fator de risco significativo para o desenvolvimento de delirium. Nesse sentido, o uso consistente e precoce de medidas de reorientação e manutenção de orientação se torna essencial para reduzir o delirium ${ }^{17}$.

A estimulação cognitiva através do livro interativo pode promover aprimoramento da memória, alerta, atenção, experiência de si, percepção e resolução de problemas, além de estimulação da concentração, 
pensamento, memória e discussões de temas atuais que podem favorecer a orientação do paciente contribuindo para prevenção de delirium em pacientes com COVID-19.

Ao desenvolver um conjunto de exercícios cujo objetivo era ativar as funções mentais, que incluem alerta, percepção visual, memória, cálculo, resolução de problemas, práxis e linguagem os pesquisadores relatam a redução de delirium após essas atividades ${ }^{13}$. As intervenções para estimulação de concentração e de aprimoramento do pensamento devem ser realizadas sempre que possível para melhorar a função cognitiva básica e podem ser desenvolvidas através de discussões sobre eventos atuais e uma estimulação da memória, promovendo auxílio terapêutico no manejo de delirium ${ }^{18}$.

O trabalho do terapeuta ocupacional com recursos bem elaborados pode ajudar no alcance dos objetivos das intervenções terapêuticas ocupacionais. Materiais como o livro interativo podem contribuir com o sentimento de apropriação do objeto ao escrever dados pessoais e materialização e organização dos seus pensamentos, tornando visível o subjetivo e podem favorecer no processo de reabilitação no que diz respeito ao manejo de delirium e ainda promover facilitação na adaptação à rotina hospitalar em pacientes com COVID-19.

O valor que o do cliente determina sobre uma tarefa influencia o nível de motivação e participação em qualquer processo de tratamento terapêutico voltado para a melhoria do desempenho ocupacional. Assim, a motivação compõe 
um importante aspecto no tratamento que pode influenciar muito no resultado funcional final, os clientes que colocam pouco valor na tarefa abordada são improváveis de chegar ao fim do tratamento para melhorar determinada habilidade ${ }^{19}$.

A intervenção terapêutica ocupacional como promotora do resgate da vida cotidiana impactada pelo adoecimento pela situação de hospitalização. Verificou também que recursos interventivos através de atividades expressivas oferecem suporte emocional emergidos pelo processo de internação e auxiliam em ações reflexivas para organização dos papéis desempenhados e reconhecimento de recursos potencializadores ao enfrentamento ${ }^{20}$.

Um estudo de estimulação cognitiva para prevenção de delirium teve como objetivo avaliar a eficácia de uma intervenção experimental não farmacológica terapêutica ocupacional na redução da incidência de delirium e afirma que as informações disponíveis sugerem a viabilidade, segurança e eficácia da terapia ocupacional na prevenção de delirium ${ }^{21}$.

\section{CONCLUSÃO}

O livro interativo é uma forma de medida não farmacológica para prevenção e manejo de delirium em pacientes hospitalizados com COVID-19. Sua metodologia promove estimulação e treinamento cognitivo junto aos pacientes hospitalizados. 
Esse estudo contribuirá como base para práticas terapêuticas ocupacionais e de pesquisadores, na elaboração de um recurso terapêutico que considere estratégias de atividades baseadas em evidências para manejo do delirium em complicações relacionadas ao COVID-19.

Visto que o delirium aumenta o tempo de hospitalização, aumentando assim os custos correspondentes, a realização de protocolos ou programas de intervenções relacionadas ao delirium junto a pacientes com COVID-19, com medidas não farmacológicas, contribuem para redução de custos e diminuição na incidência, duração, gravidade do delirium e tempo de internação prolongada.

A prevenção de delirium em pacientes com COVID-19 é condizente com os objetivos da terapia ocupacional no contexto hospitalar uma vez que ao prevenir incapacidades reduz os impactos causados pelo período de internação, mantém o desempenho ocupacional e contribui para o retorno das atividades domiciliares e comunitária com maior funcionalidade.

Esse estudo contribuirá como base para práticas de terapeutas ocupacionais e pesquisadores, recurso terapêutico com estratégias de atividades baseadas em evidências para manejo do delirium, de complicações relacionadas ao COVID-19.

Os estudos encontrados sugerem a eficácia da intervenção cognitiva e do papel da terapia ocupacional na 
prevenção do delirium, porém ainda são necessários estudos adicionais para confirmar e expandir os achados relacionados a efetividade desta intervenção junto aos pacientes com COVID-19. Os autores sugerem a realização de pesquisas e ensaios clínicos randomizados para aumentar a evidência científica dessa intervenção.

\section{REFERÊNCIAS}

1. Wu D, Wu T, Liu Q, Yang Z. The SARS-CoV-2 outbreak: What we know. Int J Infect Dis 2020;94:44-8.

http://dx.doi.org/10.1016/j.ijid.2020.03.004

2. Mao $L$, Jin $\mathrm{H}$, Wang $M, \mathrm{Hu} \mathrm{Y}$, Chen $\mathrm{S}$, He $\mathrm{Q}$, et al. Neurologic Manifestations of Hospitalized Patients with Coronavirus Disease 2019 in Wuhan, China. JAMA Neurol 2020;77:1-9.

http://dx.doi.org/10.1001/jamaneurol.2020.1127

3. Kotfis K, Williams RS, Wilson JE, Dabrowski W, Pun BT, Ely EW. COVID-19: ICU delirium management during SARS-CoV-2 pandemic. Crit Care 2020;24:176. http://dx.doi.org/10.1186/s13054-02002882-X

4. World Health Organization. ICD-10 Classification of mental and behavioural disorders: Diagnostic research criteria. Geneva: World Health Organization. 1993.

https://www.who.int/classifications/icd/en/GRNBOOK.pdf

5.Simpson R, Robinson L. Rehabilitation After Critical Illness in People With COVID-19 Infection. Am J Phys Med Rehabil 2020;99:470-4. http://dx.doi.org/10.1097/PHM.0000000000001443

6.Deemer K, Zjadewicz K, Fiest K, Oviatt S, Parsons M, Myhre B, et al. Effect of early cognitive interventions on delirium in critically ill patients: a systematic review. Can J Anaesth 2020;67:1016-34. http://dx.doi.org/10.1007/s12630-020-01670-z

7.Ribeiro MA. Design Universal. In: Souza ACA, Galvão CRC. Terapia Ocupacional: fundamentação e prática. Rio de Janeiro: Guanabara Koogan, 2007, p 417-25.

8.American Occupational Therapy Association (AOTA). Occupational Therapy Practice Framework: Domain and Process. Am J OccupTher 2014;68:S1-48. https://doi.org/10.5014/ajot.2014.682006

9. Mitchell ML, Kean S, Rattray JE, Hull AM, Davis C, Murfield JE, et al. A family intervention to reduce delirium in hospitalised ICU patients: a feasibilityrandomised controlled trial. Intensive Crit Care Nurs 2017;40:77-84. http://dx.doi.org/10.1016/j.iccn.2017.01.001

10.Alvarez E, Garrido M, González F, Guzmán E, Donoso T, Gallegos $\mathrm{S}$, et al. Terapia ocupacional precoz e intensiva enlaprevencióndel delirium en adultos mayoresingresados a unidades de paciente 
crítico. ensayo clínico randomizado: resultados preliminares. Ver Chil Ter Ocup 2012,44-59. http://dx.doi.org/10.5354/07195346.2012.22051

11.Belo AC, Cabral BMR, Lima RWF, Silva EV, Conceição RM. Atuação Do Terapeuta Ocupacional Na UTI. Rev Ter Inten 2017;1:22-9. http://www2.ebserh.gov.br/documents/210672/0/REVISTA+UTI+N\% C2\%BA+4.pdf/d115f28d-8cb5-4792-abd7-ec7e19534069

12.Stoffel DP, Nickel R. A utilização da atividade como ferramenta no processo de intervenção do terapeuta ocupacional em reabilitação neurológica. Cad Ter Ocup UFSCar 2013;21:617-22. https://doi.org/10.4322/cto.2013.064

13.Álvarez EA, Garrido MA, Tobar EA, Prieto SA, Vergara SO, Briceño $C D$, et al. Occupational Therapy for Delirium Management in Elderly Patients without mechanical ventilation in an Intensive Care Unit. A Pilot Randomized Clinical Trial. J Crit Care 2017;37:85-90. http://dx.doi.org/10.1016/j.jcrc.2016.09.002

14.Deemer K, Zjadewicz K, Fiest K, Oviatt S, Parsons M, Myhre B, et al. Effect of early cognitive interventions on delirium in critically ill patients: a systematic review. Can J Anaesth 2020;67:1016-34. http://dx.doi.org/10.1007/s12630-020-01670-z

15.Okuma SM, de Paula AFM, do Carmo GP, Pandolfi MM. Caracterização dos pacientes atendidos pela terapia ocupacional em uma unidade de terapia intensiva adulto. Rev Inte Bras Ter Ocup 2017; 1:574-88.

https://revistas.ufrj.br/index.php/ribto/article/view/8311

16. Colombo R, Corona A, Praga F, Minari C, Giannotti C, Castelli A, et al. A reorientation strategy for reducing delirium in the critically ill. Results of an interventional study. Minerva Anestesiol 2012;78:102633. $\quad$ https://www. minervamedica.it/en/journals/minervaanestesiologica/article.php?cod=R02Y2012N09A1026

17.Kersten A, Reith S. Delir und Delirmanagement bei kritisch kranken Patienten. Med Klin Intensivmed Notfmed 2016;111:1421. http://dx.doi.org/10.1007/s00063-015-0130-z

18.Zoremba N. Delirmanagement in der Intensivmedizin. Med Klin Intensivmed Notfmed 2017;320-5. https://doi.org/10.1007/s00063015-0123-y

19. Holm M, Rogers JC, James AB. Tratamentos das áreas de Terapia Ocupacional. In: Neistadt ME, Crepeau EB. Terapia Ocupacional Willard \& Spackman. Rio de Janeiro: Guanabara Koogan, 2007, p 295.

20.Bombardaa TB, Lanzab AL, Santosa CAV, Joaquima RHVT. Terapia Ocupacional na Unidade de Terapia Intensiva (UTI) adulto e as percepções da equipe. Cad Ter Ocup UFSCar 2016;24:827-35. http://dx.doi.org/10.4322/0104-4931.ctoRE0861

21.Tobar E, Alvarez E, Garrido M. Estimulação cognitiva e terapia ocupacional para prevenção de delirium. Rev Bras Ter Intensiva 2017;29:248-52. http://dx.doi.org/10.5935/0103-507X.20170034 